\title{
LA FORMACIÓN SOBRE MEDIDA: UN ELEMENTO METODOLÓGICO QUE GARANTIZA LA CUALIFICACIÓN DE LA FORMACIÓN DE DOCENTES
}

\author{
Ruth Molina Vásquez \\ Mg. Tecnologias de la Información \\ aplicadas a la Educación
}

\begin{abstract}
This paper is a summary of the final report of the graduate program "Tecnologías de la Información Aplicadas a la Educación".

The paper aims to present a reflection upon some methodological elements taken from the experience in perrnanent teachers trainings, besides to view the validity of these processeses and their perspective as qualification elements in the educational research.
\end{abstract}

\section{RESUMEN}

Este artículo es una síntesis del trabajo de tesis de la Maestría en Tecnologías de la Información Aplicadas a la Educación, donde se plantea como una reflexión en torno a los elementos que desde la experiencia se han implementado a nivel metodológico en la formación permanente de docentes, la validación de estos procesos a nivel investigativo y la perspectiva de los mismos como elementos de cualificación.

\section{RECOPILACIÓN DE EXPERIENCIAS DE FORMACIÓN DE MAESTROS}

La experiencia en los procesos de formación de docentes en tecnologías de la información aplicadas a la educación, se empieza a desarrollar al interior del Centro de Informática del Departamento de Tecnología de la Universidad Pedagógica Nacional, a partir del año de 1994. Desde ese momento se plantea como elemento metodológico un proceso en el cual se tiene en cuenta las necesidades académicas y prácticas especificas del docente, con la perspectiva de ayudar a satisfacerlas promoviendo el desarrollo social del individuo y de la comunidad a la cual pertenece, a través de la gestión de proyectos que permitan implementar las soluciones propuestas. Este proceso metodológico se denominó "formación sobre medida".

Con base en esta metodología se di-seña un proceso de formación que se aplica tanto en el Distrito Especial de Santafé de Bogotá como en Cundinamarca, a través de etapas de actualización teórica, innovación e investigación educativa (MEN, 1996), precedidas por una de diagnóstico y concertación. Este proceso parte de la concepción de pedagogía como una disciplina orientada a la solución de problemas educativos, que en este caso, se une a las tecnologías de la información, para proyectarse en resultados de cambio e innovación educativa, de que la formación permanente es un proceso continuo que se 
íntegra a todos los ámbitos de la vida cotidiana y se refleja en el aspecto académico, investigativo y de producción de conocimiento y se fundamenta en teorías que les permite a los docentes comprender los fenómenos pedagógicos y valorar procesos de construcción de conocimiento, asumir una dimensión investigativa y tecnológica para solucionar problemas específicos del ámbito educativo. Este proceso busca articular las áreas del conocimiento con la pedagogía y las nuevas tecnologías de la información a través de una estrategia intencional para generar autonomía personal e institucional, crear ambientes favorables al aprendizaje, a las transformaciones y asumir la dirección del desarrollo propio.

Desde el punto de vista teórico este modelo se fundamenta en la ciencia cognitiva, la pedagogía potenciadora y el aprendizaje significativo. El fundamento desde las tecnologías de la información, se encuentra en la utilización de procesos creativos y el diseño de la estructura y función tanto de objetos como de procedimientos orientados a resolver problemas, interviniendo la cultura para lograr su transformación hacia un estado ideal, dando como resultado una innovación tecnológica al interior de ambientes de aprendizaje, los cuales se basan en la utilización del computador y se componen de dispositivos que facilitan el acceso a la información y una interacción dinámica entre el sujeto que aprende y programa diseñado.

La estructura propuesta se aplicó en el Programas de Formación Permanente de Docentes a lo largo de cuatro años, sin embargo y pese a los resultados positivos, se empezó a configurar la necesidad de analizar y evaluar este proceso de forma más sistemática y poder comparar los resultados obtenidos con otras metodologías de formación que también parecían tener éxito. A partir de esta inquietud se plantea el proyecto de investigación titulada "Evaluación de impacto cognitivo de un modelo sobre medida en la formación permanente de docentes en tecnologías de la información aplicadas a la educación"1, la cual se plantea la siguiente pregunta: ¿Cuál de los grupos de formación permanente de docentes en tecnologías de la información, que utilizan metodología de formación sobre medida y por proyectos, hace una representación más cercana en términos de riqueza de nodos, propiedades, relaciones y construcción de proposiciones, a la red semántica sobre proyecto educativo en tecnologías de la información realizada por expertos?

\section{ELEMENTOS CONCEPTUALES PRESENTES EN LA EXPERIENCIA}

En este punto se busca caracterizar las variables incluidas en el problema planteado: Experiencias innovadoras de formación de docentes y representación de conocimiento. Inicialmente se revisan experiencias investigativas relacionadas con el tema, y curiosamente se encuentran muchas en las que se propone la utilidad de la aplicación de redes semánticas como elementos gráficos que pueden representar la estructura cognitiva de lo sujetos pero ninguna que muestre elementos de evaluación de los procesos de formación de docentes. Se encuentran experiencias valiosas en la línea de formación de docentes, en autores como René Laffitte, (Rodas, Sánchez, Orozco, Uribe, 1995-1996), Peggy Levine (Rodas, Sánchez, Orozco, Uribe, 1995-1996), Assael y Guzmán (1996), Barkatoolah (1993), Londoño, Capa, Gómez (1996), Cortés, Medina, Parra (1996) y Cardozo (1997).

\footnotetext{
${ }^{1}$ Esta investigación es realizada por la autora del presente artículo, para optar por el título de Magíster en tecnologías de la información aplicadas a la educación.
} 
Uno de los principales obstáculos para caracterizar los elementos conceptuales radicó en la diversidad de términos que se encuentran en el medio educativo como sinónimos de formación permanente: capacitación, educación continuada, educación permanente, educación de adultos, formación continuada, perfeccionamiento docente. Con base en la postura de autores como Gusdorf (1973), Imbernón (1994), UNESCO (1986), Ferry (1993) y Birgin, Dussel, Duschatzky y Tiraminti (1998), se adopta la formación permanente como aquella formación postescolar derivada de la ocupación profesional que implica la adquisición de conocimientos, capacidades, habilidades, conductas y actitudes asociadas estrechamente al campo profesional de una persona, por lo tanto necesita hacer una revisión de los elementos ya adquiridos y una renovación y actualización de estos como consecuencia de los avances de la tecnología, la ciencia y la pedagogía. Así, esta formación requiere de por lo menos cuatro componentes: la formación científica, psicopedagógica, cultural y la práctica docente. De igual manera, este proceso tomado como un elemento que genera innovación en el ámbito educativo, presenta cinco finalidades fundamentales: el mejoramiento de las competencias profesionales a nivel individual, las competencias ocupacionales dentro de los objetivos planteados institucionalmente, la ampliación de la experiencia del profesor dentro de otras funciones a cumplir en su perspectiva profesional, el desarrollo de conocimientos y la ampliación de la educación personal en general.

Por otra parte, se hace necesario caracterizar un instrumento que pueda ayudar a representar la estructura de conocimiento de los participantes en el proceso, ya que este es un aspecto de vital importancia si se quieren establecer categorías de comparación a nivel del impacto alcanzado por las dos metodologías propuestas en el problema planteado. Acudiendo a autores como Puente (1989), Shapiro (1987) y Perner (1994) se parte de la relación existente entre el procesamiento de información y memoria semántica entendida como el almacén de información acerca del conocimiento organizado del mundo y los símbolos verbales, agrupados en referentes, relaciones entre objetos, reglas, fórmulas, algorítmos y conceptos, con el objeto de realizar inferencias, generalizaciones o combinaciones de estos y procesos complejos de razonamiento. Posteriormente se hace necesario, entonces, encontrar un código simbólico que permitiera hacer la representación de la estructura cognitiva de los docentes participantes en el proceso, para lo cual se acudió a las redes semánticas, clasificadas dentro de los modelos proposicionales de representación de conocimiento, ya que están ligadas a la manera como la información es almacenada dentro de la memoria. Esta estructura representa el conocimiento como un patrón de conexiones entre nodos y arcos que muestran las relaciones entre conceptos y acontecimientos del sistema de memoria semántica, elegidos por esta a través de un proceso de reestructuración de expansión o transformación.

Con el apoyo de autores como Quillian (1969), Collins y Luftus (1975), Norman y Rumelhart (1976), Anderson (1983), Shapiro (1987), Sowa (1987), Russell y Norvig (1996) se extraen principios comunes en la elaboración de las redes semánticas y se procede a determinar los elementos que incluiría un grupo de expertos en este proceso, el cual parte de una organización jerárquica de proposiciones, compuesta por las relaciones entre conceptos y sus propiedades.

\section{3. ¿CÓMO SE EVALÚA LA EXPERIENCIA?}

Con base en lo anterior se busca comparar el nivel de impacto cognitivo que logra un modelo de formación sobre medida frente a otro que no utiliza esta estrategia. Sin embargo se planteaba una pregunta determinante para el proceso de investigación 
¿Cómo medir el impacto cognitivo logrado por los dos procesos de formación? ¿Cuáles son las categorías de comparación a tener en cuenta? Para clarificar los anteriores aspectos se acude a establecer como parámetros de evaluación el enriquecimiento o expansión de la red semántica elaborada por los participantes de los dos grupos, en términos de cantidad de nodos, propiedades, relaciones y proposiciones, elaboradas antes y después de los procesos de formación, en comparación con la red semántica elaborada por expertos.

Con ayuda de un procedimiento cuasi experimental de grupo control no equivalente (Cambell y Stanley, 1970), y del Análisis de protocolos verbales (Newell y Simon, 1972), se inició el estudio de la población, conformada por un grupo control de veinticinco docentes y por un grupo experimental con la misma cantidad de sujetos. Cabe mencionar que las características de los docentes son muy similares en cuando a aspectos como edad, nivel de formación y experiencia en el tema tratado.

Con el objeto de evaluar la riqueza de la red semántica elaborada por los docentes sometidos al proceso de formación sobre medida y por proyectos, se diseñó y desarrolló un ambiente computacianal de evaluación denominado "Construcción de redes semánticas", en cuyo modelo pedagógico se adopta una estructura compuesta por un módulo de presentación, un módulo de preguntas orientadoras; un módulo de información general, un módulo de ayuda y un módulo de evaluación en donde se plantea el problema de elaborar la red semántica. Como modelo experto se toma una red semántica elaborada por tres expertos sobre el tema de Proyectos pedagógicos en Tecnologías de la Información ${ }^{2}$, y como modelo de estudiante se busca que el usuario realice procesos y acciones como: activar objetos, los elementos que le propone al red, navegar por el software y tomar decisiones.

\section{4. ¿LA FORMACIÓN SOBRE MEDIDA TIENE IMPACTO COGNITIVO EN EL SUJETO QUE PARTICIPA?}

En los resultados de la investigación se observa que el grupo experimental, que tuvo un proceso de formación sobre medida, obtuvo en todos los aspectos, diferencias significativas con respecto al grupo control, que tuvo un proceso de formación por proyectos. Se destaca que a pesar de que los dos grupos obtuvieron ganancia en la riqueza de conceptos, propiedades, relaciones y proposiciones, estas no fueron significativas en el grupo control, mientras en el experimental sí. Por otra parte en las puntuaciones obtenidas en las pruebas iniciales, se muestra que los dos grupos partieron de las mismas condiciones pese a lo cual se vieron diferencias altamente significativas en la prueba final, a favor del grupo experimental.

Como resultado de la implementación de protocolos de información verbal, se observó que los sujetos del grupo control presentan estrategias de elaboración de la red de tipo vertical, es decir, abordaron cada uno de los elementos de construcción para todos los niveles, lo cual pudo haber generado problemas en la ubicación de nodos pues implicaba realizar el mismo proceso varias veces atendiendo a su jerarquía. Por su parte los sujetos del grupo experimental llevaron a cabo estrategias de orden horizontal, es decir, asumieron subtareas determinadas por los niveles de jerarquía más que por la estructuración de los tipos de nodos. Por ello, estos sujetos asumieron la ubicación de nodos conceptuales, de propiedades y relaciones de forma ordenada atendiendo cada

\footnotetext{
${ }^{2}$ Esta temática se constituía como el núcleo temático central de los dos procesos de formación comparados.
} 
nivel, empezando por el nivel jerárquico superior y repitiendo el mismo proceso en cada nivel jerárquico inferior deforma ordenada, lo cual se puede interpretar como una construcción más significativa de la red.

A partir de los elementos teóricos y del proceso de investigación se concluye que la metodología de formación sobre medida marca diferencias significativas en el en términos de la riqueza de la estructura cognitiva de los docentes, representada a través de una red semántica en comparación con los docentes que no utilizaron este tipo de estrategia durante su proceso de formación. Esta diferencia se marcó en la riqueza de nodos conceptuales, proposiciones, propiedades y relaciones incluidas en la elaboración de una red semántica, lo que implica que la metodología de formación sobre medida acude a elementos que activan procesos semánticos de memoria a largo plazo que permiten a los docentes participantes encontrar elementos de relación significativa entre sus necesidades particulares de formación, el proceso realizado y la estructura de su conocimiento.

Se puede afirmar que las estrategias utilizadas por los sujetos del grupo experimental acudieron a elementos de tipo significativo, que les permitieron hacer una construcción más cercana a su experiencia y mostrar que su estructura cognitiva obedece a elementos que les permite acomodar unidades significativas representadas por conceptos, propiedades, relaciones y proposiciones, los cuales son elementos característicos del manejo de una memoria de tipo semántico.

\section{5. ¿PARA DONDE VAMOS EN LOS PROCESOS DE FORMACIÓN PERMANENTE DE DOCENTES?}

Para el futuro es importante el sendero un tanto inexplorado que muestra la investigación en los procesos de formación docentes, pues abre la posibilidad de realizar un tipo de evaluación de impacto de estructuras cognitivas en los sujetos participantes en los programas de formación permanente de docentes, para determinar la eficacia de los procesos metodológicos realizados. Por otra parte también muestra una forma efectiva de aplicación de los elementos teóricos e investigativos en el ámbito de ciencia cognitiva, específicamente en la aplicación de las redes semánticas como estrategia evaluativa que busca determinar los procesos cognitivos que son favorecidos en situaciones reales de formación de docentes, ya que muestran de forma clara, procesos de estructuración de unidades de significado a través de la representación proposiconal, en las que participan elementos de acomodación, confrontación con la estructura cognitiva existente y emparejamiento de esta con una estructura vacía que se le propone al sujeto.

En el futuro, esta experiencia de evaluación de procesos de formación docente puede generalizarse hacia otras áreas del conocimiento con el objetivo garantizar la cualificación de procesos metodológicos, aunque por supuesto, genera otras inquietudes en cuanto a la ampliación del espectro hacia otro tipo de metodologías innovadoras que se proponen en el ámbito de programas de formación docente.

Experiencias de investigación como la que se sintetiza en este artículo, demuestran que los procesos de formación de docentes pueden ser evaluados y en forma muy particular, que la metodología de formación sobre medida se perfila como un elemento que ayuda al desarrollo de aprendizajes significativos por parte de los docentes participantes, aspecto que bien se puede generalizar en otros procesos de formación. Cabe mencionar en este punto, que a partir de la experiencia de los docentes en este 
proceso de formación, se puede afirmar que la implementación de proyectos pedagógicos apoyados en tecnologías de la información empieza a hacer parte del proyecto de vida de los docentes participantes, lo cual se evidencia a través de elementos como el trabajo que han seguido desarrollando los docentes en sus instituciones educativas con base en el trabajo realizado al interior del proceso de formación, su creciente participación en otros procesos deformación a nivel de posgrado y principalmente en lo que ellos mismos han llamado su incursión en la cultura tecnológica e informática.

Se podría pensar que el verdadero éxito en la cualificación de los procesos de formación de docentes radica en gran medida, en la cercanía que presenta el proceso a las necesidades que este tiene, a la configuración de soluciones factibles y reales a estas necesidades y a la posibilidad de crear y recrear de forma continua, el proceso de formación a través de una reflexión de carácter permanente que realiza el mismo docente sobre su papel como sujeto y centro de esa formación.

Lo anterior devela que de forma subterránea se empieza a configurar un modelo pedagógico abierto y flexible en torno a los procesos de formación permanente de docentes, con una clara concepción sobre la construcción de conocimiento, los procesos de aprendizaje experiencial y reflexivo desarrollados a partir de la práctica profesional y la utilización de las tecnologías de la información como un elemento que puede brindar elementos de solución a problemas educativos y a propósito de procesos investigativos y de innovación que generen autonomía personal e institucional.

\section{BIBLIOGRAFÍA}

Asaael, J., Guzmán, I. (1996). "Procesos grupales y aprendizaje en talleres de educadores: una propuesta para la formación de docentes". En: Revista Colombiana de Educación. N. 33. Santa Fe de Bogotá, Il semestre de 1996.

Barktoolah, Amina. (1993). "El aprendizaje experiencial: un nuevo enfoque para la formación y el perfeccionamiento de los docentes". En: Serie de Fundamentos de la Educación. Lectura N. 21. Santa Fe de Bogotá.

Birgil, Dussel, Dusschatzky y Tiramonti (1999). La formación docente. Argentina: Editorial Troquel S.A.

Campbell, D., Stanley, J. (1970). Diseños experimentales y cuasiexperimentales en la investigación social. Argentina: Amorrortu Editores.

Cardozo, Gilberto. (1997). "La pedagogía de la tecnología y la formación de docentes". En: Revista Educación en Tecnología. Volumen 2 N. 2. Santa Fe de Bogotá, II semestre. Pp.60-73

Ferry, Gilles. (1993). El trayecto de la formación: los enseñantes entre la teoría y la práctica. Barcelona: Editorial Paidós.

Imbernon, Francisco. (1994). La formación del profesorado. Barcelona: Editorial Paidós. semantic net versus production rule representation of subject content". En: Journan computer-based instruction. Autumn. Vol. 20, N. 4, Pp. 99-106. 
Jonassen, David., Wáng, Sherwood. (1993). "Changes in the structures Írom building

Maldonado, Luis Facundo. (1995). Análisis de protocolos: posibilidad metodológica para el estudio de procesos cognitivos en personas con discapacidad. Universidad pedagógica Nacional. Santa Fe de Bogotá. Documento de circulación interna.

Cuan, L., Gómez, R. (1996). "Proyectos educativos institucionales en informática: una estrategia de formación sobre medida para docentes en educación básica y media vocacional”. En: Acuc. Noticias. Año 24. Octubre de 1996. Págs. 32-35.

Monroy, Betty. (1995). Procesos cognitivos. Universidad Pedagógica Nacional. Documento de circulación interna. Santa Fe de Bogotá.

Perner, Josef. (1994). Comprender la mente representacional. Barcelona: Ediciones Paidós.

Puente, Anibal. (1989). Psicología cognitiva. Caracas: Editorial McGraw Hill.

Rolston, David. (1997). Principios de inteligencia artificial y sistemas expertos. Madrid: Editorial McGraw-Hill.

Shapiro, Stuart. (1987). Enciclopedia de inteligencia artificial. USA: Editorial In Chief.

Sowa, John F. (1987). "Semantics networks". En: Enciclopedia de inteligencia artificial. USA: Editorial In-Chief. Pp. 1011-1024. 